\title{
Thoracoabdominal Asynchrony Is Not Associated with Oxyhemoglobin Saturation in Recovering Premature Infants
}

\author{
Colleen Brennan ${ }^{a}$ Lara Ulm ${ }^{a}$ Samuel Julian ${ }^{a}$ Aaron Hamvas ${ }^{a}$ \\ Thomas Ferkol $^{\mathrm{a}, \mathrm{b}}$ Julie Hoffman ${ }^{\mathrm{a}}$ Laura Linneman ${ }^{\mathrm{a}}$ James Kemp ${ }^{\mathrm{a}, \mathrm{c}}$ \\ Departments of a Pediatrics and ${ }^{\mathrm{b}}$ Cell Biology and Physiology, Washington University School of Medicine, and \\ 'Sleep Medicine Laboratory, St. Louis Children's Hospital, St. Louis, MO, USA
}

\section{Keywords}

High-humidity nasal cannula $\cdot$ Prematurity $\cdot$ Respiratory

inductance plethysmography $\cdot$ Hypoxemia

\begin{abstract}
Background: Recovering premature infants are at risk for hypoxemia and lack of synchrony between their rib cage and abdomen due to airflow obstruction and poor respiratory compliance. Thoracoabdominal asynchrony (TAA) is a useful marker of resistive and elastic lung properties. Whether TAA predicts oxygenation is unknown. Objectives: We investigated oxyhemoglobin saturation $\left(\mathrm{SpO}_{2} \%\right)$ and TAA (phase angle, $\varphi$ ) in preterm infants with/without high-humidity nasal cannula (HHNC). Methods: A cross-sectional observational study was conducted in 92 infants at 32 weeks' postmenstrual age. We measured $\mathrm{SpO}_{2} \%$ with pulse oximetry and TAA with $\varphi$ via respiratory inductance plethysmography in infants (mean gestational age: $26.4+1.3$ weeks) who required room air ( $n=18$ ) or HHNC with/without supplemental oxygen (1-5 liters per minute, $\mathrm{FiO}_{2} 0.21-0.33, n=74$ ). We calculated median $\mathrm{SpO}_{2} \%$ from $24.7+10.0$ min of quiet sleep and median $\varphi$ from up to 60 breaths. Results: Infants breathing room air alone had marked TAA $\left(\varphi=83.6+32.9^{\circ}\right.$, range: 10.9-148.5) as did those receiving varying degrees of ventilatory and oxygen support via HHNC (range of group means,
\end{abstract}

$\left.\varphi=47.0-90.0^{\circ}\right)$. Infants breathing room air had statically greater median $\mathrm{SpO}_{2} \%$ than those receiving support $(96.3+$ $0.6 \%$ vs. $91.3+0.6 \%$; ANOVA $p=0.001) . \mathrm{SpO}_{2} \%$ was not associated with TAA in either group $\left(r^{2}=0.09\right)$. Conclusion: Recovering premature infants exhibited TAA regardless of need for ventilatory support and supplemental oxygen. TAA was not associated with $\mathrm{SpO}_{2} \%$ in either group. Maintenance of $\mathrm{SpO}_{2} \%$ does not require correction of TAA.

(c) 2016 S. Karger AG, Basel

\section{Introduction}

Survival in extremely premature and very low birth weight neonates has improved steadily, with $84 \%$ of all very low birth weight neonates surviving [1]. Increasingly, noninvasive technology is being used for respiratory support, with prevention of hypoxemia and increased work of breathing as its primary goals of care. Premature infants, however, have diminished lung compliance, high chest wall compliance, intermittent upper airway obstruction, and varying degrees of parenchymal lung disease [26]. While oxyhemoglobin saturation can be objectively assessed, the clinical manifestations of excessive respiratory effort are more subjective and multifactorial. Thoracoabdominal asynchrony (TAA) obtained using respiratory

\section{KARGER}

(C) 2016 S. Karger AG, Basel

E-Mail karger@karger.com

www.karger.com/neo
Colleen Brennan, MD

Division of Newborn Medicine, Department of Pediatrics

1 Children's Place, Mailbox 8116

St. Louis, MO 63110 (USA)

E-Mail Brennan_C@kids.wustl.edu 


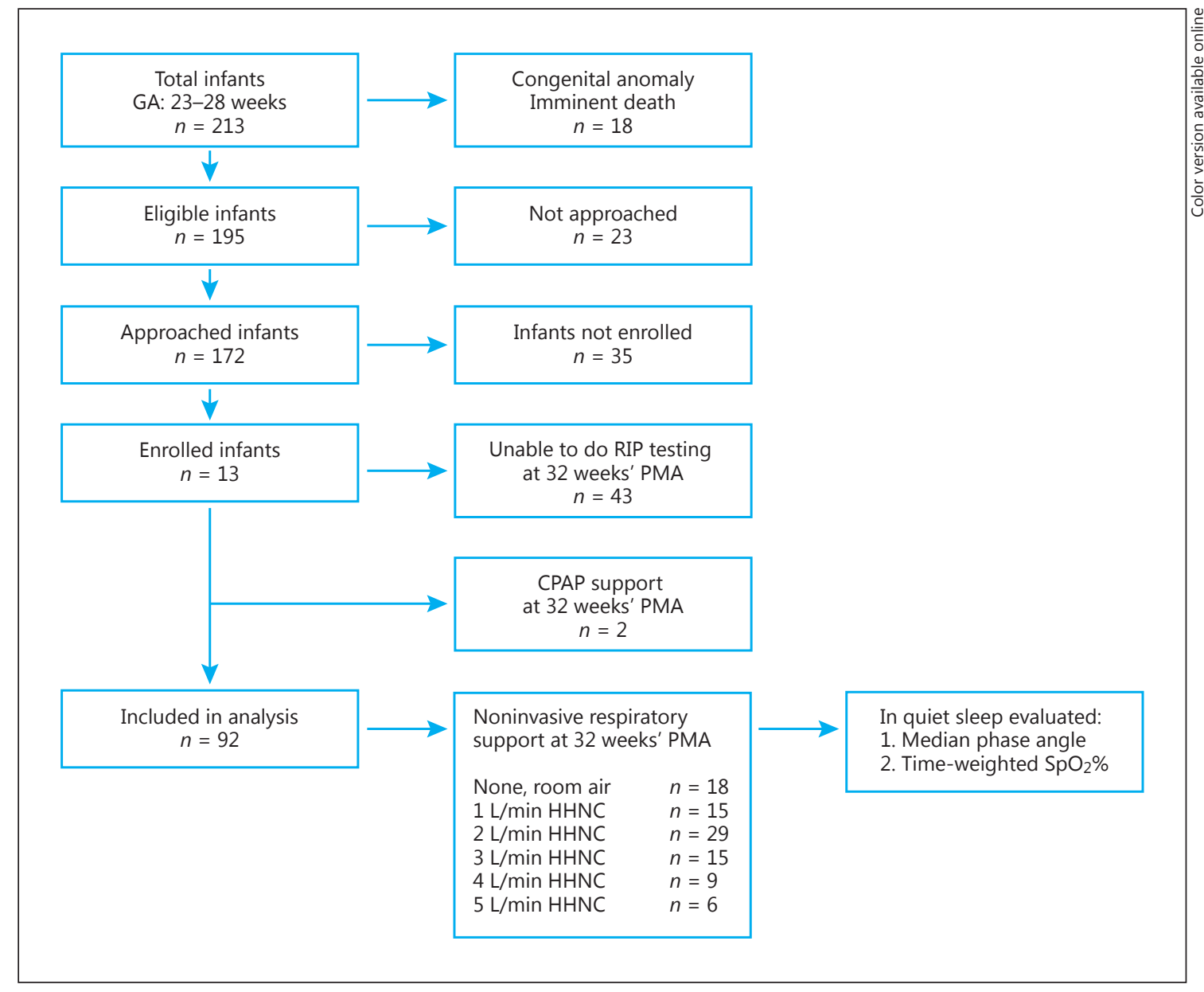

Fig. 1. The sequence for subject selection and the number of infants receiving each level of noninvasive HHNC support.

inductance plethysmography (RIP) potentially could be a marker of both resistive and elastic properties of the premature lung [7]. Whether TAA is a useful indicator of oxygen exchange in recovering preterm infants is unknown. We examined the association of oxyhemoglobin saturation $\left(\mathrm{SpO}_{2} \%\right)$ and TAA (phase angle, $\varphi$ ) in recovering preterm infants with and without noninvasive respiratory support using high-humidity nasal cannula (HHNC). We hypothesized that infants with stable oxyhemoglobin saturation $\left(\mathrm{SpO}_{2} \%\right)$ would have minimal TTA.

\section{Methods}

\section{Study Center}

These studies were conducted at Washington University School of Medicine and St. Louis Children's Hospital as a single center study within a larger multicenter collaboration (Premature Respiratory Outcome Program [PROP]; NHLBI, No. UO1HL1017).
The study protocol was reviewed and approved by the Washington University Human Research Protection Office and the PROP Observational Study Monitoring Board.

\section{Subjects}

All premature infants born at $23-28^{6 / 7}$ weeks gestation between August 12, 2010, and December 11, 2012, and hospitalized in the NICU at St. Louis Children's Hospital were screened for enrollment at birth to be studied at 32 weeks' postmenstrual age (PMA). PMA was the convention used to describe combined gestational and postnatal age. Exclusion criteria for the PROP study included known congenital anomalies or imminent death due to complications of prematurity. Families of all eligible infants admitted to the NICU were approached within 7 days of birth after acquiring permission from their attending neonatologists. Families were approached again at 32 weeks' PMA prior to testing to confirm their informed consent. All infants were spontaneously breathing and receiving no support or noninvasive respiratory support at $1-5$ liters per minute (LPM) by $\mathrm{HHNC}$. $\mathrm{FiO}_{2} \%$ varied from 0.21 to 0.50 . Both flow level and $\mathrm{FiO}_{2} \%$ settings were as prescribed by the attending neonatologist within the NICU policy of maintaining 
$\mathrm{SpO}_{2} \%<96 \%$ and $>88 \%$. We chose to study infants at 32 weeks' PMA because many infants are being treated for respiratory insufficiency via noninvasive respiratory support at that PMA $[5,6,8]$.

The sequence for subject selection and the number of infants receiving each level of noninvasive HHNC support are shown in Figure 1. Included are results from 65 infants from our earlier publication and an additional 27 subjects [8]. Infants receiving CPAP would have been an ideal comparison group to the HHNC groups; however, within our 32-week PMA cohort, only 2 subjects were on CPAP that underwent RIP, thus limiting meaningful comparison.

RIP in Infants at 32 Weeks' PMA

The phase angle $\varphi$ obtained by RIP is the measurement used in PROP to quantify TAA as an index of respiratory system function [7-9]. Breaths occurring during behaviorally determined quiet sleep (QS) were selected. QS was defined by closed eyes, regular breathing, and no fluttering of eyelids or limbs [10]. The goal for each infant was to record at least $15 \mathrm{~min}$ of QS. Measurements were obtained after a feeding with the infant supine in his/her incubator. Two RIP bands, $38.0 \mathrm{~cm}$ long unstretched and $2.0 \mathrm{~cm}$ wide, were used to record rib cage and abdominal wall excursion: one at the nipple line and one at the umbilicus. Scalar tracings from RIP were recorded in a crib-side laptop computer using proprietary hardware and software (Biocapture; Cleveland Medical Devices, Cleveland, OH, USA). The tracings were annotated every $3 \mathrm{~min}$ by an experienced sleep laboratory technician at crib side. The infant's state was labeled as QS or rapid eye movement sleep/awake.

\section{Breath Selection and Analysis}

A previously described protocol [8] was used to choose breaths for analysis. The time in QS was divided into thirds, and the first 20 acceptable breaths within each time tercile were selected. Acceptable breaths were defined by producing single closed or nearly closed Konno-Mead loops when the thoracic and abdominal wall excursion tracings were plotted against one another (loops creating a figure- 8 were not used). If a time tercile did not include 20 acceptable breaths, fewer than 20 breaths from that tercile were used. Our goal was to analyze a total of 60 acceptable breaths, 20 from the beginning of each time tercile. Of the 92 infants, 56 infants had 60 acceptable breaths during their QS analysis. The 36 infants with fewer than 60 acceptable breaths had a median of 42 breaths (range: 15-59). Scalar tracings and corresponding rib cage versus abdominal Konno-Mead plots were inspected [10]. Phase angle $\varphi$ values were calculated using VivoSense Software (Vivonoetics, San Diego, CA, USA) via the following equation: phase angle $\varphi=\sin -1(\mathrm{~m} / \mathrm{s})$.

\section{Time-Weighted $\mathrm{SpO}_{2} \%$}

We used $\mathrm{SpO}_{2} \%$ as a measure of oxygen exchange. The mean $\mathrm{SpO}_{2} \%$ for each minute of QS $(24.7 \pm 10.0 \mathrm{~min})$ was provided by the analysis software. The overall median of all $1-$ min $\mathrm{SpO}_{2} \%$ means for each infant during QS was identified. $\mathrm{SpO}_{2} \%$ was recorded continuously using a NONINR pulse oximeter (NONIN Medical Inc., Minneapolis, MN, USA). The oximeter has an effective averaging time of $1.5-3.0 \mathrm{~s}$ depending on the pulse rate.

\section{Statistical Analysis}

Demographic data, phase angle $\varphi$ for all breaths, and timeweighted $\mathrm{SpO}_{2} \%$ were described using means, SDs, medians, interquartile ranges, and coefficients of variation. All analyses were done using an IBM SPSS statistical software package (version 21; SPSS, Chicago, IL, USA). Spearman rank correlation was used to test for trends in group mean $\varphi$ at each HHNC flow rate. Differences in $\varphi$ and $\mathrm{SpO}_{2} \%$ among the groups defined by respiratory support were analyzed by ANOVA. Post hoc comparisons were done, using a Dunnett test, between $\varphi$ and $\mathrm{SpO}_{2} \%$ from infants breathing without support or with HHNC respiratory support. The relationship between $\mathrm{SpO}_{2} \%$ and $\varphi$ for each infant was explored using linear correlation. The potential effect of $\mathrm{FiO}_{2}$ on the relationship between $\mathrm{SpO}_{2} \%$ and $\varphi$, and the combined effect of $\mathrm{FiO}_{2}$ and flow rate on $\mathrm{SpO}_{2} \%$ versus $\varphi$ was analyzed using Spearman correlational coefficients with adjustment for confounders. To account for and quantify the combined effect $\mathrm{FiO}_{2}$ and flow rate, a support score was calculated as $\mathrm{FiO}_{2}$ times the flow rate. For example, an infant breathing $\mathrm{FiO}_{2}=0.40$ delivered at $2 \mathrm{LPM}$ would be assigned a "support score" of $0.40 \times 2.0=0.8$. A $p$ value of $<0.05$ was used to determine statistical significance with appropriate correction for multiple comparisons.

\section{Results}

\section{Subjects}

Table 1 summarizes subjects by demographics and level of noninvasive support received at 32 weeks' PMA: mean demographic data, duration of mechanical ventilation, $\mathrm{FiO}_{2}$, a "support correction" factor combining flow rate and $\mathrm{FiO}_{2}$, phase angles, and time-weighted $\mathrm{SpO}_{2} \%$. Coefficients of variation for $\varphi$ for all 92 subjects ranged from 9 to $123 \%$.

The groups differed by gestational age at birth (ANOVA, $p=0.006$ ), birth weight (ANOVA, $p<0.001$ ), and number of days receiving mechanical ventilation (Kruskal-Wallis test, $p=0.001)$. Post hoc comparisons of noninvasive support showed that infants receiving 2 and 3 LPM had lower gestational ages (Dunnett test, $p=0.022$ and 0.002), and infants receiving 2, 3, and 5 LPM had lower birth weights (Dunnett test vs. room air, $p=0.006$, 0.011 , and 0.022).

\section{TAA and $\mathrm{SpO}_{2} \%$}

No relationship was found between TAA as measured by phase angle and $\mathrm{SpO}_{2} \%$ (Fig. $2, r^{2}=0.09$ ). Since the amount of support an infant is receiving $\left(\mathrm{FiO}_{2}\right.$ and flow $)$ impacts saturations and work of breathing, the relationship of phase angle to amount of support being delivered was examined next, using a calculated "support correction" value (Table 1). Again no strong relationship was evident between phase angle measurement and support score alone $\left(r^{2}=0.045\right)$.

Figure 3 shows $\mathrm{SpO}_{2}$ values for infants receiving $0-5 \mathrm{~L}$ flow noninvasive support. Infants breathing without any respiratory support (zero flow) had clinically appropriate 


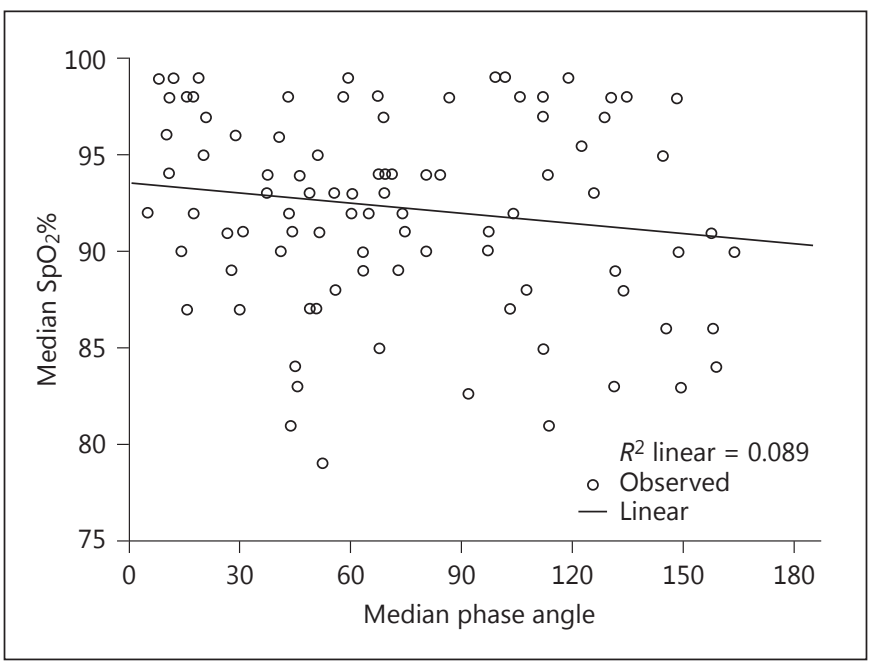

Fig. 2. For each infant, this figure shows the median $\mathrm{SpO}_{2} \%$ plotted vs. the median phase angles from the same periods of quiet sleep.

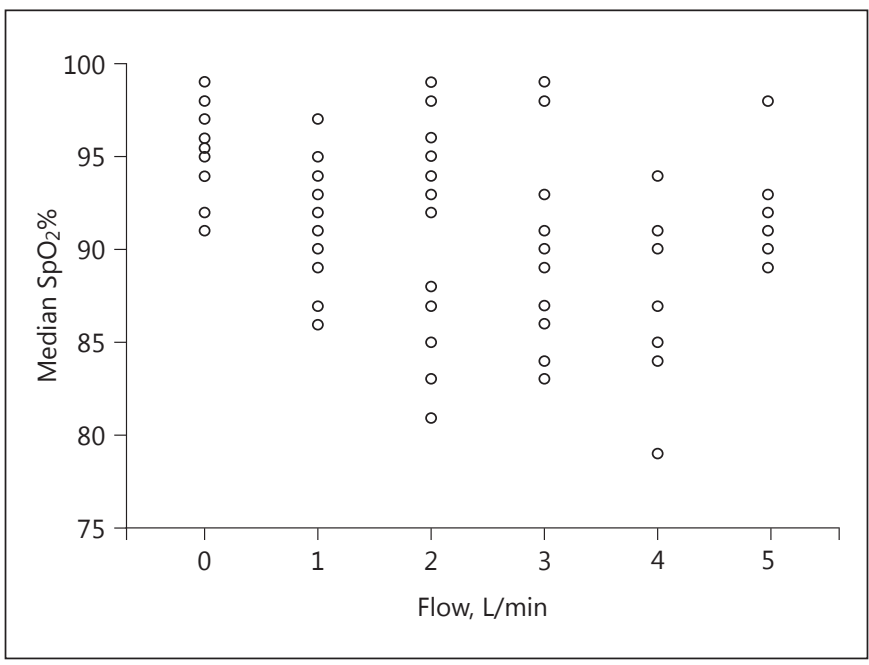

Fig. 3. $\mathrm{SpO}_{2} \%$ for each minute of quiet sleep was recorded for each infant and a median $\mathrm{SpO}_{2} \%$ identified. Each circle is the median $\mathrm{SpO}_{2} \%$ for 1 infant. There is frequent superimposition of circles for infants receiving ambient room air, and those receiving 1-4 LPM of flow.

Table 1. Clinical demographics and results

\begin{tabular}{|c|c|c|c|c|c|c|}
\hline & $\begin{array}{l}\text { No respiratory } \\
\text { support (room air) }\end{array}$ & \multicolumn{5}{|c|}{ Noninvasive HHNC support } \\
\hline Gestational age, weeks & $27.2 \pm 1.0$ & $26.8 \pm 1.4$ & $26.1 \pm 1.3$ & $25.6 \pm 1.6$ & $26.7 \pm 0.9$ & $26.0 \pm 0.6$ \\
\hline Birthweight, g & $1,059 \pm 214$ & $1,077 \pm 197$ & $866 \pm 173$ & $846 \pm 179$ & $905 \pm 250$ & $794 \pm 152$ \\
\hline Ventilator days ${ }^{\mathrm{a}}$ & 1 & 2 & 4 & 2 & 2 & 18 \\
\hline Support correction $\left(\right.$ flow $\left.\times \mathrm{FiO}_{2} \%\right)$ & 0 & $0.23 \pm 0.04$ & $0.50 \pm 0.10$ & $0.83 \pm 0.18$ & $1.26 \pm 0.44$ & $1.70 \pm 0.37$ \\
\hline Phase angle $\varphi$, degrees ${ }^{\mathrm{a}}$ & $83.6 \pm 42.5$ & $90.0 \pm 46.1$ & $69.8 \pm 43.7$ & $69.7 \pm 55.7$ & $58.6 \pm 20.1$ & $47.0 \pm 27.1$ \\
\hline $\mathrm{SpO}_{2} \%^{\mathrm{a}}$ & $96.3 \pm 2.8$ & $91.6 \pm 3.4$ & $92.1 \pm 5.3$ & $90.8 \pm 5.6$ & $88.3 \pm 5.0$ & $92.2 \pm 3.2$ \\
\hline
\end{tabular}

Data are presented as means \pm SD or $n(\%)$. ${ }^{\text {a }}$ Denotes use of medians for nonnormally distributed data.

median $\mathrm{SpO}_{2} \%$ that was $>90 \%$. There were significant differences among the median $\mathrm{SpO}_{2} \%$ for infants without support and at 5 different flow rates of HHNC (ANOVA, $p=0.001)$. The $\mathrm{SpO}_{2} \%$ of infants without support was greater than those who received 1-4 LPM of flow (Dunnett test, $p<0.001$ to $p=0.013)$, but not greater than those receiving $5 \mathrm{LPM}$ of flow (Dunnett test, $p=0.214$ ). Thus, infants breathing without respiratory support had better saturations despite greater TAA (Table 1).

Regression analysis indicates a weak correlation between increasing flow rates and decreasing median $\varphi$
(Spearman rank correlation $\left.r^{2}=0.05, p=0.036\right)$. No statistical differences were found in $\varphi$ measurements across noninvasive support groups compared to those without support (ANOVA, $p=0.279$; Dunnett test, $p=0.336-$ 0.994). When gestational age at birth and the number of days an infant required mechanical ventilation within each group were controlled, there was a suggestion that flow rates influenced differences in $\varphi$, but the results were not statistically significant (gestational age, $p=0.060$; ventilator days, $p=0.066)$. 
Differences in flow rates prescribed did not significantly alter the width of infants' $\varphi$ interquartile range, indicating that increasing flow rates did not reduce variability in an individual infant's $\varphi$ (ANOVA, $p=0.212$ ).

\section{Discussion}

Premature infants recovering from respiratory insufficiency are increasingly being treated with noninvasive high-flow nasal cannula supplemental oxygen. Evaluation of clinical markers to guide the support they receive has not previously been examined. This study is the first to quantify potential markers, like TAA, to assist in clinical decision-making. We examined time-weighted $\mathrm{SpO}_{2} \%$ and TAA during QS among 92 infants born prematurely and studied at 32 weeks' PMA. $\mathrm{SpO}_{2} \%$ was collected and averaged over $24.7 \pm 10.0$ min providing a concise and robust description of the oxygenation for all infants over the recording period. TAA measured using RIP is a welldocumented measurement of tidal breathing analysis that can detect altered lung compliance and airway obstruction $[7,10-12]$. We hypothesized that this noninvasive test could assist clinicians in their assessment of respiratory distress and hypoxemia [13-19]. TAA as measured by phase angle is not associated with oxyhemoglobin saturation; therefore, altered respiratory effort quantified using thoracic and abdominal asynchrony is not an effective predictor of $\mathrm{SpO}_{2} \%$ in this population of recovering premature infants. These results are aligned with previously reported application of TAA as a predictor of upper, rather than lower, respiratory function [20].

We have also shown that infants breathing without respiratory support had $\mathrm{SpO}_{2} \%$ that were $>90 \%$ and acceptable for clinicians directing their care, despite phase angles that were considerably greater than those previously recorded in other "well" premature infants $\left(38-60^{\circ}\right)[10]$. The $\mathrm{SpO}_{2} \%$ of these infants without respiratory support was significantly higher than infants supported by HHNC.
This observation may result from our standard NICU approach of keeping $\mathrm{SpO}_{2} \%$ in the defined range of $88-96 \%$ when delivering supplemental oxygen and flow in order to limit the potential for oxygen toxicity in preterm infants [21]. While this approach in part explains the lower $\mathrm{SpO}_{2} \%$ for infants receiving support, it does not confound or detract from our findings that infants without respiratory support had stable oxyhemoglobin saturations despite much TAA. Correction of TAA is not needed to maintain adequate oxygenation.

A limitation of this study is its observational nature. We did not systematically decrease flow rates among infants using $\mathrm{HHNC}$, in order to test the hypothesis that flow provided by $\mathrm{HHNC}$ improves TAA or $\mathrm{SpO}_{2} \%$. While these reductions in $\mathrm{FiO}_{2}$ and flow are often performed at 36 weeks' PMA to categorize the presence of BPD, such a study is less feasible in infants at 32 weeks' PMA when they are still recovering from their respiratory disease. A better option for future studies might be to record $\varphi$ and $\mathrm{SpO}_{2} \%$, as flow rates are increased according to a stepwise protocol for infants with greater TAA [14].

In summary, this study demonstrates that maintenance of $\mathrm{SpO}_{2} \%$ does not require correction of TAA in premature infants recovering from respiratory insufficiency. The degree of TAA alone does not appear to be a reliable indicator of underlying pathology.

\section{Disclosure Statement}

The authors were supported by the National Institutes of Health (NIH) award HL101465, which supports the Prematurity and Respiratory Outcomes Project (PROP). The views expressed do not necessarily reflect the official policies of the Department of Health and Human Services, nor does mention by trade names, commercial practices, or organizations imply endorsement by the US government. The authors have no other financial relationships or other conflicts of interest that compromise, or may be perceived to compromise, the integrity of the observations herein. All subjects' parents gave written informed consent for infant participation. All research complies with guidelines for human studies.

\section{References}

1 Lemons JA, Bauer CR, Oh W, Korones SB, Papile LA, Stoll BJ, et al: Very low birth weight outcomes of the National Institute of Child health and Human Development Neonatal Research Network, January 1995 through December 1996. NICHD Neonatal Research Network. Pediatrics 2001;107:E1.

Thoracoabdominal Asynchrony and Oxyhemoglobin Saturation
2 Holleman-Duray D, Kaupie D, Weiss MG: Heated humidified high-flow nasal cannula: use and a neonatal early extubation protocol. J Perinatol 2007;27:776-781.

-3 Manley BJ, Owen LS, Doyle LW, Andersen CC, Cartwright DW, Pritchard MA, Donath SM, Davis PG: High-flow nasal cannulae in very preterm infants after extubation. $\mathrm{N}$ Engl J Med 2013;369:1425-1433.
4 Kubicka ZJ, Limauro J, Darnall RA: Heated, humidified high-flow nasal cannula therapy: yet another way to deliver continuous positive airway pressure? Pediatrics 2008;121:82-88.

$\checkmark 5$ Mayfield S, Jauncey-Cooke J, Hough JL, Schibler A, Gibbons K, Bogossian F: Highflow nasal cannula therapy for respiratory support in children. Cochrane Database Syst Rev 2014;3:CD009850. 
6 Yoder BA, Stoddard RA, Li M, King J, Dirnberger DR, Abbasi S: Heated, humidified high-flow nasal cannula versus nasal CPAP for respiratory support in neonates. Pediatrics 2013;131:1482-1490.

-7 Allen JL, Greenspan JS, Deoras KS, Keklikian E, Wolfson MR, Shaffer TH: Interaction between chest wall motion and lung mechanics in normal infants and infants with bronchopulmonary dysplasia. Pediatr Pulmonol 1991; 11:37-43.

8 Ulm L, Hamvas A, Ferkol TW, Rodriguez OM, Cleveland CM, Hoffman JA, Sicard-Su MJ, Kemp JS: Sources of methodological variability in phase angles from respiratory inductance plethysmography in preterm infants. Ann Am Thorac Soc 2014;11:753-760.

9 Allen JL, Sivan Y: Measurements of chest wall function; in Stocks J, Sly PD, Tepper RS, Morgan WJ (eds): Infant Respiratory Function Testing. New York, John Wiley and Sons, 1996, pp 344-349.

10 Grigg-Damberger M, Gozal D, Marcus CL, Quan SF, Rosen CL, Chervin RD, Wise M, Picchietti DL, Sheldon SH, Iber C: The visual scoring of sleep and arousal in infants and children. J Clin Sleep Med 2007;3:201-240.

11 Warren RH, Horan SM, Robertson PK: Chest wall motion in preterm infants using respiratory inductive plethysmography. Eur Respir J 1997;10:2295-2300.

12 Heldt GP, McIlroy MB: Distortion of chest wall and work of diaphragm in preterm infants. J Appl Physiol 1987;62:164-169.

13 Shaffer TH, Greenspan JS, Alapati D, Wolfson MR: Neonatal non-invasive respiratory support: physiological implications. Pediatr Pulmonol 2012;47:837-847.

14 Spence KL, Murphy D, Kilian C, McGonigle R, Kilani RA: High-flow nasal cannula as a device to provide continuous positive airway pressure in infants. J Perinatol 2007;27:772-777.

15 Elgellab A, Riou Y, Abbazine A, et al: Effects of nasal continuous positive airway pressure (NCPAP) on breathing pattern in spontaneously breathing premature newborn infants. Intensive Care Med 2001;27:1782-1787.

16 Chang GY, Cox CA, Shaffer TH: Nasal cannula, CPAP, and high-flow nasal cannula: effect of flow on temperature, humidity, pres- sure, and resistance. Biomed Instrum Technol 2011;45:69-74.

17 Dysart K, Miller TL, Wolfson MR, Shaffer TH: Research in high flow therapy: mechanisms of action. Respir Med 2009;103:14001405.

18 Hasan RA, Habib RH: Effects of flow rate and airleak at the nares and mouth opening on positive distending pressure delivery using commercially available high-flow nasal cannula systems: a lung model study. Pediatr Crit Care Med 2011;12:29-33.

19 LeSouef PN, England SJ, Bryan AC: Total resistance of the respiratory system in preterm infants with and without an endotracheal tube. J Pediatr 1984;104:108-111.

20 Hammer J, Newth CJ, Deakers TW: Validation of the phase angle technique as an objective measure of upper airway obstruction. Pediatr Pulmonol 1995;19:167-173.

21 Support Study Group of the Eunice Kennedy Shriver NICHD Neonatal Research Network, et al: Target ranges of oxygen saturation in extremely preterm infants. N Engl J Med 2010; 362:1959-1969. 